\title{
L'utilisation des sols par le jardinage urbain à grande échelle de Russie
}

The use of grounds by large-scale gardening of Russia

\section{Louiza Boukharaeva et Marcel Marloie}

\section{(2) OpenEdition}

\section{Édition électronique}

URL : http://journals.openedition.org/economierurale/3317

DOI : $10.4000 /$ economierurale.3317

ISSN : 2105-2581

\section{Éditeur}

Société Française d'Économie Rurale (SFER)

\section{Édition imprimée}

Date de publication : 21 octobre 2011

Pagination : 176-191

ISSN : 0013-0559

\section{Référence électronique}

Louiza Boukharaeva et Marcel Marloie, «L'utilisation des sols par le jardinage urbain à grande échelle de Russie », Économie rurale [En ligne], 325-326 | septembre-décembre 2011, mis en ligne le 17 octobre 2013, consulté le 19 avril 2019. URL : http://journals.openedition.org/economierurale/3317 ; DOI : 10.4000/economierurale.3317 


\section{L'utilisation des sols par le jardinage urbain à grande échelle en Russie}

Louiza BOUKHARAEVA • Réseau international « Développement durable des villes : le rapport entre l'urbain et la nature ", Fondation maison des sciences de l'homme, Paris louiza.boukharaeva@msh-paris.fr

Marcel MARLOIE • Équipe Proximités UMR SADAPT AgroParisTech ; marcel.marloie@live.fr

\section{Introduction}

a catégorie « jardinage urbain »n'existe Lpas dans la nomenclature des activités économiques de l'INSEE en France (Baudelet, 2009), d'Eurostats au niveau de l'Union européenne, ni de la FAO au niveau mondial. Cette absence pose problème dans un monde de plus en plus urbanisé, dans lequel le besoin d'accès à la nature apparaît fondamental pour la personne humaine. Aujourd'hui les critiques se développent contre l'étalement urbain et le modèle pavillonnaire qui détruisent les sols et accroissent l'empreinte écologique des urbains. La recherche s'oriente vers l'étude des sols des villes et de leurs périphéries (Chevery, Gascuel, 2009). L'étude du jardinage urbain de Russie nous fournit des références pour inventer de nouveaux modèles d'accès des urbains aux sols et à la nature qui soient plus durables sur le plan social et environnemental ${ }^{1}$.

Le jardinage urbain est largement répandu en Europe du Nord, du Centre et de l'Est. Mais c'est en Russie qu'il est le plus développé. Il peut être qualifié de jardinage urbain à grande échelle $e^{2}$ si on considère que cette activité est pratiquée par plus de la

1. Cet article est rédigé dans le cadre d'une recherche intitulée : «L'utilisation des sols urbains et périurbains pour le développement durable des villes », et soutenue financièrement par le Programme GESSOL du ministère français de l'Écologie, du Développement Durable, des Transports et du Logement (MEDDTL).

2. Qualificatif employé par Alasheev et al., (2000), pour désigner une partie de cette agriculture urbaine et de ses fonctions : «large-scale use of subsistence plots by city residents ». moitié des urbains sur des surfaces variant de 300 à plus $1000 \mathrm{~m}^{2}$.

C'est une agriculture urbaine familiale ${ }^{3}$ au sens d'une activité extra-professionnelle pratiquée par des citadins non-agriculteurs. L'essentiel de la production est destiné à l'autoconsommation. Les concepts de secteur informel ou d'économie souterraine sont inappropriés puisqu'elle est institutionnalisée. C'est une partie de l'économie familiale et de l'économie domestique.

Hors de Russie, des aspects importants de cette activité sont étudiés sous l'angle de l'histoire des datchas et des collectifs de jardins, potagers et datchas ${ }^{4}$ (Lowen 2003 ; Traven 2005). Des spécialistes de l'agriculture y font référence (Hervé, 2001). Des articles de journaux en révèlent l'importance (Naudet, 1997). Quelques travaux d'institutions internationales nous révèlent sa dimension. Ainsi, un rapport du Programme des Nations Unies pour le développement (PNUD) (Smith et al.,1996) indique que $65 \%$ des familles moscovites étaient engagées dans l'agriculture urbaine en 1991, contre seulement $20 \%$ en 1970 et

3. L'apport du jardinage urbain russe à la théorisation de l'agriculture urbaine est développé dans un autre article (Boukharaeva, Marloie, 2010).

4. Le mot datcha vient du verbe davat (donner), se référant au don de terres par l'État (processus et résultat de l'action de donner). C'est une petite propriété de terre qui, depuis Ivan IV dit « Le Terrible » (1530-1584), était donnée comme cadeau par le Tsar où en résultat d'un partage juridique. Aujourd'hui, ce terme recouvre deux significations : 1. la maison de campagne avec son espace de terre ; 2 . une catégorie de jardins avec leur habitation dans les collectifs de datchas. 
que la proportion était plus élevée dans les villes de province, atteignant $80 \%$ en Sibérie. En 2000, une note (United Nations Division for Sustainable Development, 2000) évaluait à $50 \%$ la part de la population russe ayant accès à de petites parcelles de terres données aux citadins, lesquelles auraient produit $30 \%$ du total de l'alimentation du pays. Les publications à caractère sociologique et anthropologique ont débattu de la manière d'évaluer leur rentabilité, de leur rôle dans la phase de transition à l'économie de marché, des relations de réciprocité qui s'y établissent (Clarke, 2002 ; Zavisca 2003 ; Ortar, 2005 ; Hervouet, 2006 ; Southworth, 2006). La plupart décrivent la multifonctionnalité de ces espaces, l'importance relative des fonctions de production alimentaire et des fonctions de plaisir et de repos. Mais il n'existe pas de vision globale de ce phénomène, ni sur le plan statistique, ni quant à sa signification pour la personne et la société.

En Russie, les témoignages des organisateurs des collectifs ${ }^{5}$ de jardins (Bassine, 1958), les informations des revues de jardinage (Jardins attenants, Maître chez soi, Vos six ares), les travaux de recherche cidessus mentionnés permettent de comprendre l'ampleur, le caractère intégral et la qualité particulière de cette activité.

Au début des années 2000, le jardinage urbain et l'avenir des collectifs donnaient lieu à des interprétations diverses. Une question fut rapidement tranchée à savoir que l'accroissement de leur production dans les années 1990 n'était pas le signe d'un retour des urbains à leurs pratiques paysannes anciennes (Alasheev et al., op. cit.). C'était le résultat de la nécessité, c'est-à-dire de la

\footnotetext{
5. Nous utilisons les expressions « collectifs de jardins », « collectifs de potagers » et « collectifs de datcha » pour désigner les collectifs en tant qu'institution juridique. Nous utilisons les expressions « jardins collectifs », « potagers collectifs » et « datchas collectives » pour désigner les espaces collectifs et les parcelles qui les composent.
}

pénurie due à la chute de la production des kolkhozes et des sovkhozes, du chômage provoqué par l'effondrement des industries, de la baisse considérable des revenus, notamment dans l'enseignement où le pouvoir d'achat fut divisé par trois à cinq et où les salaires furent payés avec des mois de retard ${ }^{6}$.

Le jardinage urbain n'est donc pas un héritage du passé rural de la Russie, condamné à péricliter avec l'adaptation des anciens paysans au mode de vie urbain. Mais n'est-ce pas plutôt un phénomène spécifique au système soviétique, et qui serait appelé à disparaître dans la nouvelle société en construction? Après une parenthèse de soixante-dix années, les villes de la Russie nouvelle retrouveraient ainsi la trajectoire universelle tracée par les villes d'Europe de l'Ouest et des États-Unis.

Les deux premières parties de cet article montrent qu'il n'en est rien. Le développement de cette activité s'apparente en fait à une double conquête sociale, le droit à la propriété de la terre et le droit à la seconde habitation. Ceci explique en grande partie le dynamisme avec lequel ce secteur d'activité s'est développé dans les années 1990 et se recompose dans la période actuelle. La troisième partie montre que face à l'effondrement de la production des entreprises agro-industrielles et au développement très lent du nouveau secteur des fermiers individuels, le secteur dit de l'économie de la population a considérablement accru sa production, ce qui a permis de surmonter la crise alimentaire des années 90. La partie suivante rend compte de l'importance quantitative du jardinage urbain au sein de cette économie de la population. Nous soulignons ensuite que la crise alimentaire se terminant, cette activité se recompose en valorisant davantage ses fonctions d'espaces de villégiature. La dernière partie rend compte du mouvement actuel d'institutionnalisation des

6. Témoignages recueillis lors des enquêtes. 
collectifs de jardins, potagers et datchas qui regroupent la plus grosse partie de ce jardinage, et constituent aujourd'hui un acteur spécifique.

Les données statistiques, en particulier celles du recensement agricole de Russie de 2006 (Service fédéral de la statistique d'État de la Fédération de la Russie, 2008) permettent de savoir comment évoluent depuis 1990 les productions de ce jardinage, les surfaces qui lui sont consacrées, le nombre des collectifs et de leurs parcelles ${ }^{7}$. Beaucoup sont inédites hors de Russie ce qui justifie de les mettre à disposition des lecteurs sous la forme de tableaux.

Les données qualitatives résultent des études conduites dans les années 2000. Après une exploration préliminaire dans les lopins paysans et les jardins urbains au cours de la période 1998-2001, une enquête dans une vingtaine de jardins collectifs de la ville de Kazan (1 200000 habitants) a été réalisée en 2002. Cinq terrains d'investigations ont été ouverts de 2003 à 2007 et suivis chaque année jusqu'à aujourd'hui. Quarante entretiens avec des jardiniers ont été réalisés en 2003 et 2004 en utilisant la méthode ethnosociologique du « récit de vie » (Bertaux, 1997). Quinze témoignages approfondis de jardiniers ont ensuite été recueillis par le moyen d'un concours organisé en 2005 avec la revue de jardinage de la ville de Kazan Maître chez soi. Les acteurs associatifs et institutionnels ont été identifiés et interrogés. Une quinzaine d'interviews d'experts ont été conduites dans l'administration, les entreprises, les associations, les revues de jardinage. En août 2010, une enquête a été conduite auprès de quarante-huit présidents d'associations

7. La parcelle désigne le jardin, le potager ou autre espace de terrain détenu par une famille. Chaque parcelle est communément appelée Sad (jardin). Ses utilisateurs se dénomment Sadovodi (jardiniers). La taille peut varier de 300 à $1500 \mathrm{~m}^{2}$ pour les jardins collectifs. La majeure partie est de $600 \mathrm{~m}^{2}$, d'où l'expression chest sotok (six ares), qui les désigne dans des revues de jardinage. régionales ${ }^{8}$ de collectifs de jardins, potagers et datchas ayant participé à Saint-Pétersbourg à un congrès de l'Union des jardiniers de Russie. Elle porte sur les tendances de l'évolution du jardinage urbain, les relations entre les associations et les pouvoirs publics, le rôle potentiel du jardin collectif dans le développement des villes et dans l'aménagement des territoires. La recherche a été complétée par l'analyse des articles des revues de jardinage ci-dessus mentionnées et des données juridiques publiées en Russie.

\section{La conquête de la terre}

Sans entrer de manière approfondie dans la question de la terre en Russie, rappelons qu'elle a toujours été cruciale, complexe et même dangereuse. La fusion séculaire du pouvoir d'État et de la propriété symbolisait la stabilité politique de la société. Dans les mentalités et l'expérience concrète de la société, l'État et le pouvoir incarnaient en même temps l'unité du territoire. La propriété de la terre fut constamment une prérogative de cet État. Aussi, l'expression «propriété privée de la terre » désignait la propriété d'une couche sociale au service du pouvoir. La quasi-totalité de la population ne pouvait pas en posséder. Les paysans, à savoir la partie la plus importante de cette population, disposaient de l'usage d'un lopin de terre dans un régime de servage qui les a attachés à la glèbe à partir de 1649, et qui les a maintenus dans une situation d'insécurité et d'asservissement. Cette histoire a fait l'objet de plusieurs travaux dont une importante rétrospective en langue française (Marxer, 2003).

8. Le mot régional désigne ici les unités politiques et administratives de la Fédération de Russie. Elles sont au nombre de 89 . Le congrès de l'Union des jardiniers de Russie a réuni 500 délégués des collectifs de jardins, potagers et datchas parmi lesquels 63 présidents d'associations régionales regroupant ces collectifs. 
La propriété privée s'est développée avec la formation d'une «bourgeoisie » à partir de la seconde moitié du XIXe siècle. Chez les paysans, l'abolition du servage en 1861 puis la réforme de Stolypine après la révolution de 1905 permirent l'émergence d'une paysannerie libre qui commençait elle aussi à accéder à la propriété. Mais ces processus ont été détruits par les politiques mises en œuvre à la suite de la révolution d'octobre 1917. Depuis la fin des années 1980, le droit à la propriété de la terre a été réaffirmé, notamment par la loi de février 1990 sur les nouveaux « Fondements de la législation foncière de l'URSS et des républiques », et est inscrit dans la constitution de la nouvelle fédération de Russie (loi du 12 décembre 1993).

Mais à la fin des années 1990, la privatisation des terres en milieu rural ne répondait pas aux espoirs de ses promoteurs. En même temps, les investigations réalisées en république du Tatarstan de 1998 à 2000 suggéraient que les urbains étaient plus favorables que les ruraux à obtenir la propriété pleine et entière de leurs parcelles, en dépit des difficultés administratives et financières.

L'une des raisons de la volonté des urbains de posséder une parcelle de terre tient au fait qu'une grosse partie de la population citadine actuelle s'est formée dans le contexte particulier de la collectivisation forcée, de la dékoulakisation et de l'industrialisation des années 1920 et 1930. Les paysans migrants en ville savaient travailler le sol, gardaient le mode de vie et les conceptions du monde, hérités de leur situation antérieure. Mais les privations de la Seconde Guerre mondiale et la famine de 1948 sont peut-être davantage explicatives car elles sont plus récentes et ont marqué la majeure partie de la population urbaine. L'aspiration à une parcelle de terre pour garantir sa sécurité alimentaire et améliorer ses conditions de vie est le fait d'une majorité d'urbains de toutes origines.
Les résultats des enquêtes montrent que les sols de ces parcelles ne sont pas simplement considérés comme des espaces pour planter. Ils sont transformés en valeurs culturelles comme le révèle le langage utilisé. L'aspiration à l'indépendance, à la sécurité, à l'autonomie est bien exprimée par le titre de la revue de jardinage Maître chez soi. Le caractère identitaire et patrimonial de la parcelle de jardin transparaît dans les entretiens par des expressions telles que : «Mes six ares de chair et de sang ». L'attachement dont les sols sont l'objet se manifeste aussi de diverses manières qui rappellent ce que l'ancêtre paysan qualifié de koulak disait : "Ceci est à moi, dans ma poigne »; "Je ne laisse pas partir cette chose qui m'appartient ».

Ces perceptions culturelles du sol expliquent que le nombre de jardins et autres types de parcelles ait été fortement accru comme il est montré dans cet article, pardelà le fait qu'un certain nombre de jardins collectifs furent détruits et certaines parcelles abandonnées dans la crise consécutive à la fin de l'Union soviétique. Elles expliquent aussi les transformations apportées à ces sols. Les terrains concédés étaient généralement inutilisables par les kolkhozes et les sovkhozes. Ils étaient souvent de mauvaise qualité, dans des endroits vallonnés ou marécageux. Les urbains les ont aménagés, amendés et transformés en sols aujourd'hui classés par l'Administration fiscale comme étant plus fertiles que les terres agricoles (entretien avec Vassili Zakhariachev, président de l'Union des jardiniers de Russie, août 2010).

Ainsi, le jardinage urbain n'est pas un phénomène éphémère, spécifique au système soviétique, et appelé à disparaître. C'est une conquête contre l'héritage du servage et de la collectivisation forcée, contre l'insécurité alimentaire des périodes de crise. Il en résulte que la Russie devient l'un des pays du monde où la proportion d'urbains propriétaires d'une parcelle de terre est la plus importante. 


\section{La conquête de la double habitation}

Dans les collectifs de jardins et de datchas, chaque parcelle comporte une habitation, une remise pour les outils, et souvent un sauna. La remise à outil fut toujours autorisée. En revanche, le sauna et l'habitation résultent d'une conquête progressive à l'intérieur du système soviétique. Le sauna était parfois dissimulé sous l'apparence d'une maisonnette. Mais c'est l'habitation qui a suscité et provoque encore de multiples débats.

Tout en acceptant la création de collectifs de jardins et de potagers, le pouvoir soviétique combattait la construction d'une maisonnette sur les parcelles. Ainsi, un arrêté du Soviet des ministres de l'URSS du 3 juillet 1952 autorisait ces constructions seulement au-delà de $50 \mathrm{~km}$ de la ville. Il interdisait de nouvelles constructions entre 10 et $50 \mathrm{~km}$, ordonnait leur destruction au-dessous de $10 \mathrm{~km}$. Cette politique fut poursuivie après la mort de Staline. Mais les populations n'ont pas cessé de ruser avec les directives pour conquérir ce droit, jusqu'à ce que la loi du 18 mars 1966 autorise ces constructions. En 1967, le passage à la semaine de cinq jours permet deux jours de repos consécutifs par semaine, ce qui ouvre la possibilité de dormir au jardin. L'État continue de freiner l'utilisation de la maisonnette en tant que seconde habitation. Elle ne doit pas dépasser $25 \mathrm{~m}^{2}$, et la véranda $12 \mathrm{~m}^{2}$. Il est interdit d'ouvrir une mansarde, de construire une cheminée, de bétonner (Traven, op. cit.).

L'arrivée au pouvoir de Mikhaïl Gorbatchev marque une rupture. Tout en affirmant qu'il faut créer les conditions pour que toute personne désirant un jardin puisse l'obtenir, il accroît la liberté de construction en permettant dès décembre 1985 d'augmenter la surface de la maisonnette à $50 \mathrm{~m}^{2}$ au sol, sans compter les surfaces des vérandas et des locaux de services (remise à outils, sauna).

L'effondrement du régime soviétique ouvre une nouvelle période, décrite en détail par nombre des personnes rencontrées lors de nos enquêtes. Dès la fin de l'année 1992, la taille maximum d'une parcelle peut atteindre $1500 \mathrm{~m}^{2}$ et une même famille peut en posséder plusieurs. Il n'y a plus de limite à la taille des maisons. Il devient donc possible de regrouper des parcelles pour y construire des maisons de deux ou trois étages. Un certain nombre de jardins collectifs se transforment progressivement en zones d'habitations. À Saint-Pétersbourg, le comité de construction et d'architecture a mis au point un nouveau plan d'occupation du territoire qui transforme les jardins collectifs en collectifs de constructions d'habitations individuelles. Ce plan permettrait aux propriétaires d'une parcelle d'y construire leur habitation principale. Selon Vassili Zakhariachev, la ville encourage les investissements pour y implanter les services collectifs, y compris des écoles.

En de multiples endroits, une pression s'exerce de la part de certains urbains pour établir leur domicile principal dans leur parcelle de jardin ou de datcha. Cette possibilité refusée par loi fédérale sur le jardinage collectif urbain a fait l'objet d'une requête au tribunal constitutionnel de Russie de la part d'un groupe de sept jardiniers de la région de Krasnodar. Le jugement émis leur a donné raison en avril 2008 en les autorisant à inscrire leur domicile dans leur maison de jardin à la condition que celle-ci réponde aux normes d'un logement habitable salubre.

Le combat se poursuit. Le ministre du Développement régional de Russie vient de proposer d'interdire de construire des maisons sur les parcelles à partir de 2015. Et les jardiniers réunis au sein de l'Union des jardiniers de Russie se mobilisent actuellement contre cette proposition.

Dans le périurbain des grandes villes, en particulier de Moscou, une partie des nouvelles installations s'écarte donc du concept antérieur de jardins collectifs. Leur population, essentiellement composée de « nouveaux russes ${ }^{9}$ est moins diversifiée 
Tableau 1. Évolution des principales productions agricoles (en millions de tonnes)

\begin{tabular}{l|r|r|r|r|r|c}
\hline & 1992 & 1998 & $1999 / 2001$ & $\mathbf{2 0 0 3 / 2 0 0 4}$ & $\mathbf{2 0 0 6}$ & $\mathbf{2 0 0 7}$ \\
\hline Total céréales & 103,8 & 46,9 & 67,2 & 70,9 & 76,9 & 80,5 \\
\hline - dont blé & 46,2 & 27,0 & 37,5 & 39,7 & 45,0 & 49,4 \\
- dont céréales secondaires & 56,9 & 19,5 & 29,2 & 30,7 & 31,2 & 30,4 \\
\hline Betteraves à sucre & 25,5 & 10,8 & 14,6 & 20,4 & 30,0 & 29,0 \\
\hline Total viande & 8,3 & 4,7 & 4,4 & 5,0 & 5,2 & 5,6 \\
\hline Lait & 47,2 & 33,3 & 32,5 & 32,8 & 31,4 & 32,2 \\
\hline Fufs & 2,4 & 1,8 & 1,9 & 2,0 & 2,1 & 2,1 \\
\hline Pommes de terre & 38,2 & 31,4 & 35,4 & 36,3 & 38,6 & 36,8 \\
\hline
\end{tabular}

Source : FAOSTAT - Site WEB

que celle des jardins collectifs, et dispose d'un revenu élevé. Les maisons sont généralement de taille importante. Ces installations gardent le même statut juridique que d'autres collectifs de jardins, mais sont qualifiées de « villages de cottages » et présentent les caractéristiques des zones pavillonnaires à la périphérie des villes occidentales.

Mais cette évolution vers le modèle pavillonnaire est freinée par les faibles revenus d'une partie de la population. Selon Vassili Zakhariachev, $80 \%$ des familles disposant d'une ou plusieurs parcelles dans les jardins collectifs ont des revenus inférieurs ou égaux à la moyenne de la Russie et n'ont pas la possibilité financière de construire des maisons luxueuses. Seuls le peuvent les $10 \%$ considérés comme riches et les $10 \%$ comme très riches. De plus l'évolution vers le modèle pavillonnaire est limitée par le fait que la plupart des urbains veulent continuer de vivre en ville, et d'utiliser leur jardin comme un espace de villégiature.

Le jardinage urbain reste donc massif. Les sols de la majeure partie des collectifs et de leurs parcelles demeurent sans bitume ni béton et sont l'objet de pratiques culturales qui préservent ou améliorent leur fertilité. Les acteurs de cette forme de jardinage sont à la recherche du statut juridique permettant à ces collectifs de ne pas évoluer vers le modèle pavillonnaire en répondant aux besoins de

9. Membres de la nouvelle classe de personnalités d'affaire, constituée depuis 1990. sécurité alimentaire et de villégiature de la plupart des familles urbaines.

Mais il est d'ores et déjà possible d'affirmer que la Russie présente cette particularité d'être un des pays du monde où la démocratisation de l'accès des urbains aux sols et à la nature par le jardinage et la double habitation est la plus avancée. Cette conquête sociale résulte des aspirations ancestrales d'une grande partie du peuple russe, celle de la possession de la terre et celle du prestige attaché au mythe de la datcha.

\section{Crise des années 1990 Aide alimentaire et croissance de l'économie de la population}

Environ les trois quarts de la valeur de la production agricole étaient assurés jusqu'en 1990 par les entreprises agro-industrielles : kolkhozes, sovkhozes et entreprises agricoles dépendant de l'industrie et de l'armée ${ }^{10}$. Ces entreprises étaient confrontées à la privatisation des années 1990 (Jogolena, Kovalenko, 1995), à la baisse du soutien financier de l'État, à la libéralisation des prix, à la hausse des coûts des intrants (Giroux, 1995) puis à l'obsolescence de leur équipement. La crise qui en est résultée s'est concrétisée par une baisse drastique de

10. La statistique officielle comptabilise aujourd'hui 27800 unités d'une surface moyenne de 11868 ha, 20400 d'une surface moyenne de 3736 ha, et 11000 exploitations auxiliaires d'entreprises non agricoles, le tout regroupant de 410,3 millions d'hectares. 
Tableau 2. Part des trois sous-secteurs dans la valeur de la production agricole

\begin{tabular}{|c|c|c|c|c|c|c|c|c|}
\hline & 1990 & 1995 & 2000 & 2002 & 2005 & 2006 & 2007 & 2008 \\
\hline $\begin{array}{l}\text { Entreprises } \\
\text { agro-industrielles }\end{array}$ & 73,7 & 50,2 & 43,4 & 39,8 & 41,2 & 41,2 & 47,5 & 48,1 \\
\hline Fermiers & & 1,9 & 3,0 & 3,7 & 5,6 & 6,5 & 8,1 & 8,5 \\
\hline $\begin{array}{l}\text { Économie de la } \\
\text { population (lopins } \\
\text { paysans et jardinage } \\
\text { des urbains) }\end{array}$ & 26,3 & 47,9 & 53,6 & 56,5 & 53,2 & 52,3 & 44,4 & 43,4 \\
\hline
\end{tabular}

leurs productions, et par conséquent de la production agricole de la Russie. En 1998, le volume des récoltes par rapport à 1992 était à $59 \%$ pour le blé, à $34 \%$ pour les céréales secondaires à $42 \%$ pour les betteraves sucrières et à $57 \%$ pour les viandes (tableau 1).

Dans l'hiver 1990-1991, les menaces de pénurie avaient déjà provoqué une aide alimentaire internationale. De nouveau, la baisse de la production et les effets du krach financier de la mi-août 1998 suscitèrent dans les médias occidentaux des rumeurs de risques de famines (Giroux, 1999). Les États-Unis répondirent en proposant en novembre 1998 la livraison de 1,7 million de tonnes de blé, de soja, de riz, de viande de bœuf et de porc, de poudre de lait et de produits divers. Confrontée à l'accumulation de ses propres stocks de produits agricoles, l'Union européenne offrit pour sa part de livrer 1,5 million de tonnes de céréales, 100000 tonnes de viande de porc, 150000 tonnes de viande de bœuf et 50000 tonnes de lait en poudre. Ces propositions suscitèrent des polémiques concernant le montant réel des réserves, la rétention possible d'une partie de la récolte par les producteurs, les utilisations pour l'alimentation animale et la stratégie alarmiste des lobbies commerciaux pour faire monter les prix.

La baisse de la production de blé était certes importante. Mais l'aide n'apparaissait nécessaire que ponctuellement pour remédier à des situations d'urgence. La population en difficulté fut évaluée par la Croix Rouge à 1,7 million de personnes. Pour sa part, le Gouvernement canadien a fourni 1,6 million de dollars pour acheter des denrées alimentaires sur place et les distribuer (Giroud, 1999). Cette aide fut mieux acceptée.

Car les diagnostics internationaux de la

Tableau 3. Part des entreprises agro-industrielles dans les volumes de production agricole

\begin{tabular}{l|c|c|c|c|c|c}
\hline & 1980 & 1990 & 2000 & 2005 & 2007 & 2008 \\
\hline Grains & 99,6 & 99,7 & 90,7 & 80,6 & 78,8 & 78,1 \\
\hline Betteraves sucrières & 100 & 99,99 & 94,4 & 88,4 & 87,9 & 89,2 \\
\hline Graines de tournesol & 97,9 & 98,6 & 84,4 & 72,5 & 69,9 & 70,7 \\
\hline Pommes de terre & 34,6 & 33,9 & 6,5 & 8,4 & 10,1 & 11,4 \\
\hline Légumes & 67,2 & 69,9 & 22,0 & 18,7 & 18,9 & 19,2 \\
\hline Bétail et volailles & 70,2 & 75,2 & 40,2 & 46,2 & 51,2 & 54,3 \\
\hline Lait & 73,1 & 76,2 & 47,3 & 44,9 & 44,3 & 44,0 \\
\hline CEufs & 73,2 & 78,4 & 70,9 & 73,7 & 74,4 & 74,6 \\
\hline Laine & 80,5 & 75,5 & 37,5 & 25,7 & 21,2 & 18,4 \\
\hline Miel & 42,3 & 30,6 & 9,7 & 5,6 & 5,0 & 4,6 \\
\hline
\end{tabular}

Source : Annuaires statistiques de la Fédération de Russie, 2002 et années suivantes 
RECHERCHES

Louiza BOUKHARAEVA, Marcel MARLOIE

Tableau 4. Part de l'économie de la population dans les volumes de production agricole

\begin{tabular}{l|c|c|c|c|c|c}
\hline & 1980 & 1990 & 2000 & 2005 & 2007 & 2008 \\
\hline Pommes de terre & 65,4 & 66,1 & 91,2 & 88,8 & 85,5 & 83,5 \\
\hline Légumes & 32,8 & 30,1 & 74,7 & 74,4 & 72,4 & 70,7 \\
\hline Bétail et volailles & 29,8 & 24,8 & 58,0 & 51,4 & 45,9 & 42,7 \\
\hline Lait & 26,9 & 23,8 & 50,9 & 51,8 & 51,7 & 51,7 \\
\hline CFufs & 26,8 & 21,6 & 28,7 & 25,6 & 24,8 & 24,5 \\
\hline Laine & 19,5 & 24,5 & 56,8 & 54,7 & 55,7 & 55,4 \\
\hline Miel & 57,7 & 69,4 & 88,2 & 91,6 & 91,6 & 92,0 \\
\hline
\end{tabular}

Source : Annuaires statistiques de la Fédération de Russie, 2002 et années suivantes

situation russe se fondaient sur une fausse interprétation. Pour s'en rendre compte, il convient d'effectuer la distinction entre les trois grands sous-secteurs de la production alimentaire, qui ont réagi très diversement à la crise (tableau 2).

En raison de la baisse drastique de sa production, la part des entreprises agroindustrielles est passée de $73,7 \%$ en 1990 à $39,9 \%$ de la production agricole totale en 2002. Elle a ensuite progressivement augmenté pour représenter 48,1\% en 2008. Comme l'indique le tableau 3, ces entreprises continuent de jouer un rôle prépondérant dans les productions de grains, de betteraves sucrières, de tournesols et d'œufs. Mais leur part des productions de pommes de terre, de légumes, de laine, de miel est très faible.

Le sous-secteur des fermiers individuels était composé en 2008 de 267000 unités cultivant 29,4 millions d'ha soit une surface moyenne de 103 ha. Inexistante en $1990^{11}$, leur production devient significative en particulier pour les grains $(21 \%$ de la production totale), les betteraves sucrières

11. La collectivisation forcée avait éliminé les agriculteurs indépendants. Les changements sous Gorbatchev, puis la loi de février 1990 sur les nouveaux «Fondements de la législation foncière de l'URSS et des Républiques » donnent le droit de posséder la terre à vie et de la transmettre en héritage. Cette loi permet aussi aux membres des kolkhozes et sovkhozes de les quitter avec une parcelle de terre qui vient s'ajouter à leur lopin traditionnel.
$(9,8 \%)$, le tournesol $(28,9 \%$, les légumes $(10,1 \%)$, la laine $(26,2 \%)$.

Le sous-secteur le plus dynamique fut celui qualifié « d'économie de la population $^{12} »$. Sa part de la valeur de la production agricole a plus que doublé entre 1990 et 2002, passant de 26,3 à $56,5 \%$ pour régresser à $43,4 \%$ en $2008^{13}$. Le tableau 4 montre qu'il assure l'essentiel des productions de pommes de terre (83,5\% en 2008) et de légumes $(70,7 \%)$, ainsi qu'une proportion notable des bétails et viandes, lait, laine et miel.

12. L'Annuaire statistique de Russie de 2009 (notes méthodologiques, Moscou, Service fédéral des statistiques d'État) indique que «L'économie de la population comprend les lopins et les autres économies individuelles des citoyens dans des zones rurales et urbaines, ainsi que les terres dans les unions non commerciales de jardins, potagers et datchas».

13. Les données de 1980 à 2008 sont collectées et traitées par des méthodes identiques ou comparables. Depuis 2006, elles tiennent compte des résultats du recensement agricole de 2006. Les données sont issues des enquêtes auprès des membres des collectifs de jardiniers, potagers et datchas par la méthode de l'observation statistique sélective. Un échantillon sélectif des parcelles est créé par échantillonnage aléatoire systématique. La sélection se base sur une liste des parcelles à l'exclusion des abandonnées ou pas installées. L'enquêté est le propriétaire ou l'utilisateur, in Service fédéral de la statistique d'État de la Fédération de la Russie, 2008. 
Tableau 5. L'économie de la population en 2008

\begin{tabular}{l|c|c|c}
\hline & Nombre en milliers & $\begin{array}{c}\text { Superficie totale } \\
\text { en milliers d'ha }\end{array}$ & $\begin{array}{c}\text { Superficie } \\
\text { moyenne en } \text { m }^{2}\end{array}$ \\
\hline Total & 40454 & 9459 & 2338 \\
\hline dont : lopins & 16037 & 7076 & 4400 \\
\hline dont : jardinage urbain & 24417 & 2383 & 975 \\
\hline dans les jardins & 14004 & 1249 & 890 \\
\hline dans les datchas & 121 & 25 & 2066 \\
\hline dans les potagers & 2977 & 300 & 1008 \\
\hline dans les construction & & 809 & 1106 \\
\hline d'habitations individuelles & 7315 & & \\
\hline
\end{tabular}

Source : À partir de l'Annuaire statistique 2008 de la Fédération de Russie

\section{Place du jardinage urbain dans l'économie de la population}

Le tableau 5 présente les diverses catégories de terrains répertoriés sous cette rubrique « économie de la population ». Ce sont en premier lieu les lopins dont le nombre varie autour de seize millions dans toute la période allant de 1990 à aujourd'hui. Il s'agit essentiellement de parcelles d'environ $4000 \mathrm{~m}^{2}$, adjointes aux maisons individuelles des villageois, incluant un espace de potager, ainsi que des cultures permettant l'existence d'un petit élevage (cheval, porc, volailles). Une fraction estimée par l'Union des jardiniers de Russie à environ deux millions de parcelles est localisée en zones urbaines et périurbaines et peut être comptabilisée dans le jardinage urbain.

Ces seize millions de lopins occupent la majeure partie des surfaces de l'économie de la population, mais les autres catégories représentent un nombre supérieur de parcelles (24,4 millions en $2008-c f$. tableau 6). Il s'agit des jardins, des datchas, des potagers collectifs et des constructions d'habitations individuelles, pratiquement tous utilisés par des urbains. Certaines familles possèdent plusieurs parcelles. L'Union des jardiniers de Russie estime à 21 millions le nombre de familles urbaines dans ces collectifs ${ }^{14}$.

14. Entretien avec Vladimir Deïnekin vice-président de cette Union et président de l'Association des collectifs de jardins de Kazan.
Si l'on retient le coefficient de 2,7 personnes par ménage (recensement de 2002), cela signifie que 56,7 millions d'urbains Russes utilisent une parcelle dans ces collectifs. Pour avoir une idée plus complète de l'ensemble du jardinage urbain, il faut ajouter un nombre non répertorié de lopins en zones urbaines et périurbaines (environ 2 millions) ainsi que les jardins individuels en dehors des collectifs. On peut alors estimer que le jardinage urbain concerne entre 56 à 70 millions d'urbains soit 54 à $67 \%$ d'une population urbaine estimée à 104200000 personnes. C'est près du double de la population rurale [38 millions], et plus de quatre fois la population agricole [13,5 millions].

Les données résultant d'enquêtes conduites au niveau des villes confirment cette importance. À Moscou, l'Institut de géographie estime que $50 \%$ des moscovites ont accès à un jardin, un potager ou une datcha ${ }^{15}$. À Saint-Pétersbourg et sa région, la proportion est plus élevée selon l'Union des jardiniers de Russie ${ }^{16}$. Elle l'est encore plus dans les villes de province. Par

15. Interview de Vladimir Kolossov, professeur à l'Institut de géographie de l'Académie des sciences de Moscou, novembre 2008.

16. Interview de Vassili Zakhariachev. En 2004, la Direction des jardins et potagers comptabilisait 600000 parcelles de jardins individuels, 120000 parcelles de potagers, et 180000 lopins. 
RECHERCHES

Louiza BOUKHARAEVA, Marcel MARLOIE

Tableau 6. Le jardinage urbain : évolution du nombre de parcelles et de surfaces (en millions de parcelles et en milliers d'hectares)

\begin{tabular}{|c|c|c|c|c|c|c|c|}
\hline \multirow{3}{*}{$\begin{array}{l} \\
\text { Jardins individuels } \\
\text { et dans les collectifs }\end{array}$} & & 1990 & 1995 & 1998 & $2001 / 2004$ & 2007 & 2008 \\
\hline & Nombre & 8,5 & 15,0 & 15,1 & 14,6 & 14,0 & 14,0 \\
\hline & Superficie & 600 & 1200 & 1300 & 1300 & 1200 & 1200 \\
\hline \multirow{2}{*}{$\begin{array}{l}\text { Datchas } \\
\text { individuelles et } \\
\text { dans les collectifs }\end{array}$} & Nombre & 0,12 & 0,12 & 0,08 & 0,09 & 0,11 & 0,12 \\
\hline & Superficie & 18 & 18 & 12 & 13 & 25 & 25 \\
\hline \multirow{2}{*}{$\begin{array}{l}\text { Construction } \\
\text { d'habitations } \\
\text { individuelles }\end{array}$} & Nombre & & 5,0 & 6,1 & 6,6 & 7,2 & 7,3 \\
\hline & Superficie & & 500 & 600 & 700 & 800 & 800 \\
\hline \multirow[t]{2}{*}{ Sous total } & Nombre & 8,62 & 20,12 & 21,28 & 21,29 & 21,31 & 21,42 \\
\hline & \begin{tabular}{|l} 
Superficie \\
\end{tabular} & 618 & 1718 & 1912 & 2013 & 2025 & 2025 \\
\hline \multirow{2}{*}{$\begin{array}{l}\text { Potagers individuels } \\
\text { et dans les collectifs }\end{array}$} & Nombre & 5,1 & 7,4 & 7,0 & 4,6 & 3,0 & 3,0 \\
\hline & Superficie & 400 & 600 & 600 & 400 & 300 & 300 \\
\hline \multirow[t]{2}{*}{ Total } & Nombre & 13,72 & 27,52 & 28,28 & 25,89 & 24,31 & 24,42 \\
\hline & Superficie & 1018 & 2318 & 2512 & 2413 & 2325 & 2325 \\
\hline
\end{tabular}

Source : Annuaires statistiques de la Fédération de Russie et Agence fédérale du cadastre, rapport 2008

exemple, le Centre d'informations régionales de Irkoutsk (580 000 habitants) estime que $75 \%$ des adultes de cette ville disposent d'un jardin ou d'un potager.

Dans les années 1990, les rumeurs de famines dans les médias occidentaux résultaient pour partie du fait que, à l'exception des deux documents ci-dessus mentionnés (le rapport du PNUD de 1996 et une note de la United Nations Division for Sustainable Development en 2000) les statistiques publiées par les institutions internationales ignoraient ce jardinage urbain.

Ainsi notamment, un rapport de l'OCDE indiquait que : «En 1997, seize millions de lopins familiaux se partageaient environ 5,7 millions d'hectares..., leur part dans la production agricole totale a considérablement progressé depuis le début de la transition pour se situer à $50 \%$ de la production agricole brute $(P A B)$ en $1997 »$ (OCDE, 1998). En fait, ce n'étaient pas 16 millions de lopins familiaux, identifiés dans le rapport à des lopins paysans, qui assuraient ces $50 \%$ de la production agricole brute. C'étaient au total environ 44 millions de parcelles, la majorité étant exploitée intensivement par des urbains.

\section{Une transformation qualitative du jardinage urbain}

Lors de la crise des années 1990, le nombre de jardins, potagers et datchas s'est considérablement accru, passant de 13,7 millions en 1990 à 22,2 millions en 1998 pour une surface d'environ 1,9 million d'hectares (tableau 6). En même temps une catégorie nouvelle est apparue, les constructions d'habitations individuelles. Ainsi qu'il a été précédemment mentionné, il s'agit de terrains sur lesquels il est autorisé de construire une vraie maison, alors que les maisonnettes sur les jardins devaient auparavant correspondre à des critères limitant leur surface et leur hauteur. Ces constructions d'habitations individuelles restent entourées d'une parcelle de sol et contribuent à la production alimentaire. Elles sont construites dans des jardins créés antérieurement ou sur de nouveaux terrains. Inexistantes en 1990 elles sont au nombre de cinq millions en 1995 et de 7,5 en 2008. La statistique publique les comptabilise dans la catégorie des parcelles individuelles (tableau 8).

Au total, les jardins, potagers, datchas et constructions d'habitations individuelles ont atteint 28,3 millions en 1998, pour régresser 


\section{Encadré 1. Jardins, potagers, datchas}

Les définitions précises données aux collectifs de jardins, potagers et datchas dans le rapport du Comité d'État du cadastre du 1er janvier 2008 permettent de comprendre les différences qualitatives entre ces diverses appellations.

Les collectifs de potagers ont pour but : «La culture de petits fruits, de légumes, de cucurbitacés et autres cultures agricoles avec ou sans droit de bâtir une construction habitable légère avec ses dépendances. » On perçoit ici que les potagers restent des lieux de production potagère (les arbres fruitiers ne sont pas mentionnés) en ouvrant la possibilité d'y construire une construction habitable légère avec ses dépendances qui peut permettre d'en faire un espace modeste de villégiature.

La définition du jardin fait moins de place aux productions potagères et reconnaît le droit d'en faire des lieux de villégiature plus effectifs : «Les collectifs de jardins ont pour but la production des cultures fruitières et de petits fruits, le repos, avec le droit de construire une maison d'habitation et ses dépendances. »

La définition des collectifs de datchas est davantage centrée sur les fonctions de villégiature tout en mentionnant le droit à la production : «Elles ont pour but le repos, avec le droit de bâtir une construction habitable ou une maison, et avec le droit de planter et de produire diverses cultures agricoles. »

Dans la réalité, les différences entre collectifs de datchas et de jardins ne sont pas toujours visibles. La majorité des Russes ne parlent pas de leur datcha mais de leur potager (Ogorod) ou de leur jardin (Sad). Leur seconde habitation s'appelle maisonnette (domik) ou maison (dom).

à 24,4 en 2008. Ces données chiffrées rendent compte d'une évolution qualitative de l'accès des urbains à leur parcelle de sol. Espaces uniquement destinés à la production alimentaire, les potagers ont fortement régressé, passant de 7 à 3 millions. Le nombre des autres catégories de terrains, qui sont à la fois des espaces de production alimentaire et de villégiature, est resté stable (tableau 6).

Le nombre de jardins a pour sa part baissé d'un million ce qui recouvre plusieurs phénomènes. Un certain nombre de jardins ont été abandonnés, d'autres ont été créés, et une partie est passée dans la catégorie des constructions d'habitations individuelles. Au total, les jardins, datchas et constructions d'habitations individuelles sont passés de 21,3 millions en 1998 pour une surface de 1,9 millions d'hectares à 21,1 millions en 2008 pour une surface de deux millions d'hectares. Leur surface moyenne s'est légèrement accrue. Ainsi qu'il a été précédemment mentionné, il faudrait ajouter à ces chiffres de 2008 un nombre non répertorié de lopins en zones urbaines et périurbaines, de même que les terrains achetés de manière croissante par les urbains en zones rurales.
Les présidents des quarante-huit associations régionales de collectifs de jardins de la Fédération de Russie attestent que les besoins des urbains d'accéder à un jardin, un potager ou une datcha sont satisfaits en termes quantitatifs, que l'évolution va dans le sens d'une amélioration qualitative, par une diversification des usages de ces terrains. Dans la plupart d'entre eux, la place réservée aux pommes de terre régresse. Il reste presque toujours un espace réservé aux légumes de qualité et de convivialité (tomates et cornichons notamment) aux arbres et arbustes fruitiers et aux fraises. Les gazons sont plus nombreux ainsi que les espaces récréatifs. Ce sont de plus en plus des espaces de villégiature au sens où ils remplissent les fonctions d'extension de l'habitat urbain, de récupération de la santé, d'éducation des enfants, de convivialité, de loisirs, de créativité.

\section{L'institutionnalisation des collectifs}

Le mode d'organisation de ce jardinage en fait aujourd'hui une institution sociale qui compte autour de 79900 collectifs de potagers, jardins, et datchas (chiffres de 2006, 
RECHERCHES

Louiza BOUKHARAEVA, Marcel MARLOIE

Tableau 7. Les collectifs de jardins, datchas et potagers en 2006

\begin{tabular}{l|c|c|c|}
\hline Unions commerciales de : & $\begin{array}{c}\text { Nombre d'unions } \\
\text { (en milliers) }\end{array}$ & $\begin{array}{c}\text { Superficie } \\
\text { (en millions d'ha) }\end{array}$ & $\begin{array}{c}\text { Superficie par union } \\
\text { (en ha) }\end{array}$ \\
\hline - jardinage & 73,3 & 1,12 & 15 \\
\hline - datchas & 1,1 & 0,02 & 18 \\
\hline - potagers & 5,5 & 0,07 & 12 \\
\hline Total & 79,9 & 1,21 & 15 \\
\hline
\end{tabular}

Source : Annuaire statistiques de la Fédération de Russie - 2007

(tableau 7). La personnalité juridique leur a été attribuée par la loi fédérale du $1^{\mathrm{er}}$ avril 1998 sur les unions non commerciales citoyennes de jardins, de potagers et de datchas. Plusieurs statuts sont définis : camaraderies jardinières non commerciales ; coopératives de consommation ; partenariats non commerciaux ${ }^{17}$.

La taille de ces collectifs peut varier énormément et compter de quelques dizaines à plusieurs milliers de parcelles. Selon les responsables des associations de jardinage interrogés en août 2010, leur nombre irait en s'accroissant. Presque toutes les catégories de la population y sont présentes, telles que directeurs d'usine, ouvriers, employés de bureaux, médecins, chauffeurs de taxi, universitaires.

À Kazan, l'Association des jardins collectifs a identifié environ 700 collectifs de jardiniers, potagers et datchas. Ils regroupent 160000 parcelles couvrant au total près de dix mille hectares.

Le modèle du collectif de jardins existait dès 1918 , et quelques-uns avaient été créés

17. « Les unions non commerciales citoyennes de jardins, potagers et datchas (camaraderie non commerciale de jardinage, de potager ou de datcha ; coopérative de consommateurs non commerciale de jardinage, de potager ou de datcha ; partenariat non commercial de jardinage, de potager ou de datcha) sont des organisations sans but lucratif fondées par des citoyens sur une base volontaire pour aider leurs membres dans la résolution des tâches communes sociales et économiques du jardinage, du potager et de datcha (Article 1er de la loi fédérale du 15 avril $1998 n^{\circ}$ 66-FZ « sur les unions non commerciales de citoyens de jardins, potagers et datchas »). dans les années 1920 et 1930. Le pouvoir soviétique n'y était pas favorable au nom du dogme selon lequel les fermes collectives allaient satisfaire tous les besoins. Mais la désorganisation de l'économie, les destructions provoquées par la guerre, les pénuries alimentaires et les famines obligèrent le Gouvernement central à encourager le jardinage urbain. Selon la Grande Encyclopédie soviétique, 18,5 millions d'urbains travaillaient la terre en 1945 contre 5 millions en 1942. Les sols agricoles cultivés en zones urbaines et périurbaines étaient passés dans le même temps de 1,4 million d'ha à 5 millions. Voulant que cette activité fonctionne selon les principes socialistes, les responsables de l'État utilisèrent le modèle des collectifs. Ils furent l'objet d'une intense activité législative. L'analyse des documents législatifs nous a permis d'identifier environ 400 lois, amendements et décrets édités à leur propos depuis 1950.

Leur création a été autorisée par un arrêté du 4 novembre 1942. Il organisait « la domiciliation des parcelles de terre au sein de l'entreprise et l'organisation de potagers individuels octroyés aux travailleurs et aux employés ». Les impôts sur la production agricole des ouvriers et des employés furent supprimés le 19 juin 1943 (Traven, 2005). Les entreprises et les institutions pouvaient s'adresser au syndicat et au Soviet des villes pour recevoir un terrain qu'elles devaient aménager. Ce mécanisme est resté le même jusqu'à la fin des années 1980.

Chaque collectif de jardins était géré par une entité de type association ou coopérative au sein des entreprises, mais qui n'avait pas la personnalité juridique. Les grandes déci- 
Tableau 8. Le jardinage urbain : surfaces plantées en 2006 (en hectares)*.

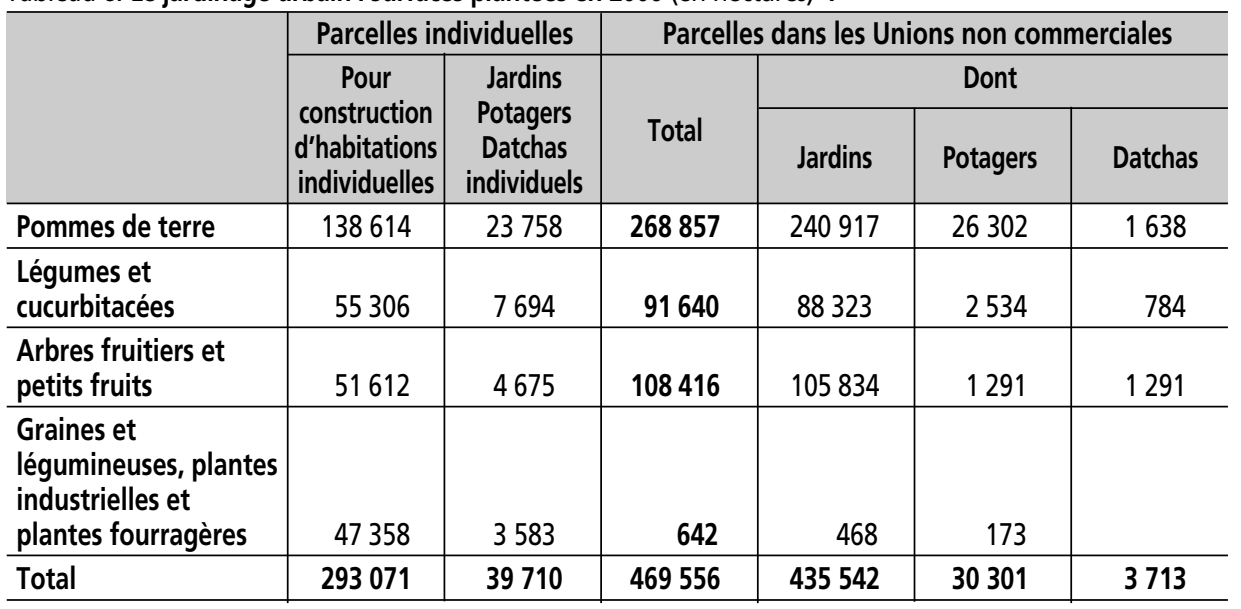

Source : Réalisé à partir des tableaux 12, 15 et 18 de l'annuaire statistique de la Fédération de Russie, 2008

$\left(^{*}\right)$ Sans compter les lopins et autres terrains des urbains en zones périurbaines et rurales

sions leur échappaient. Leur financement était assuré par l'entreprise. Avec la privatisation, ces dernières n'ont plus été en mesure d'entretenir les collectifs, et les pouvoirs publics n'ont pas pris le relais dans la plupart des villes. Aussi, ces entités se sont souvent retrouvées seules face aux problèmes d'entretien des espaces communs, des lignes électriques et des canalisations d'eau, d'évacuation des déchets, d'accès aux transports en commun, d'insécurité.

Elles ont été poussées par la force des évènements à devenir de véritables associations décidant de leurs orientations, élisant et contrôlant leurs responsables. Cette réalité a fini par être intégrée par le système législatif.

Les bouleversements des vingt dernières années se sont traduits par la disparition de certains collectifs, et par l'apparition de nouvelles installations. À partir du début des années 2000 ont été créés des ensembles qualifiés de «macif », gardant le statut des collectifs de jardins, mais s'identifiant progressivement à des villages ruraux modernes. Ils sont particulièrement nombreux dans la banlieue de Saint-Pétersbourg, de préférence localisés dans des endroits ayant un certain prestige. Ils peuvent inclure un magasin, un restaurant, un complexe de loisir.
Le tableau 8 montre qu'en 2006, les surfaces plantées dans ces Unions non commerciales étaient de 469556 hectares dont 435542 hectares dans les jardins. Elles fournissaient $14,3 \%$ des pommes de terre, $18,3 \%$ des légumes et cucurbitacées, $29,1 \%$ des fruits et petits fruits de toute l'économie de la population de Russie.

Le processus de privatisation n'a donc pas provoqué la destruction de cette forme d'organisation. Elles restent des espaces collectifs gérés par les unions non commerciales, qui édictent et font respecter les règles de fonctionnement, veillent à l'entretien des chemins, clôtures et autres services communs, représentent les intérêts de la collectivité et peuvent agir en justice. Au sein de ces unions, les jardiniers peuvent accéder à la propriété de leur parcelle. Amorcée à la fin des années 1980 cette privatisation a consisté à les donner gratuitement et en pleine propriété à ceux qui les utilisaient. En 2007 le pourcentage des parcelles privatisées varie de 5,9\% pour les potagers à $77,9 \%$ pour les datchas (tableau 9). Les différences tiennent au fait que les familles les plus aisées, qui disposaient d'une datcha, ont plus facilement surmonté les coûts de la procédure d'enre- 
gistrement et la complexité administrative. Les plus modestes ayant besoin d'un potager pour subsister se sont résignés au statu quo.

Tableau 9. Part des parcelles privatisées en 2007

\begin{tabular}{l|r}
\hline Lopins & $31 \%$ \\
\hline Jardins & $33,6 \%$ \\
\hline Potagers & $5,9 \%$ \\
\hline Datchas & $77,9 \%$ \\
\hline Constructions d'habitations individuelles & $45,9 \%$ \\
\hline
\end{tabular}

Source : Agence fédérale du cadastre, rapport 2008

La loi fédérale $n^{\circ} 93$ du 30 juin 2006 sur « la simplification de l'enregistrement des droits des biens immobiliers » constitue une étape importante. Entrée en vigueur le $1^{\text {er }}$ septembre 2006, elle est qualifiée par la population de «amnistie des jardins » au sens où ses nouvelles règles facilitent la privatisation des parcelles. Un Service fédéral d'enregistrement, du cadastre et de la cartographie a été créé le $1^{\text {er }}$ mars 2009 pour simplifier leur enregistrement.

\section{Conclusion}

Si la catégorie « jardinage urbain » était introduite dans les nomenclatures des appareils statistiques nationaux et internationaux, la connaissance de la situation alimentaire des pays serait plus exacte, et les réponses aux crises plus adaptées.

Bien que résultant d'une histoire spécifique à la Russie, le jardinage urbain à grande échelle de ce pays présente un intérêt universel. Les 79900 collectifs de jardins, potagers et datchas, aujourd'hui sujets de droit, constituent une institution sociale spécifique, un acteur urbain du travail sur les sols et la nature unique au monde.

Il ne s'agit pas de la réminiscence du passé rural, ni d'un phénomène éphémère spécifique à la période soviétique. Pour des populations qui héritent des bouleversements de la collectivisation, de l'industrialisation et de l'urbanisation forcées, des répressions, des déportations, de la guerre et des famines, la possession d'un jardin est un symbole de stabilité, d'accès à un mode de vie apaisé. Elle s'apparente à la construction d'une société nouvelle.

Le développement de ce jardinage au cours des années 1990 était pour partie une réponse à une conjoncture de crise. Mais c'est surtout une double conquête, celle de la propriété de la terre refusée à l'époque du servage puis sous la période soviétique, et celle du droit à utiliser le jardin comme une extension de l'habitat urbain en y construisant une maisonnette où il soit possible de résider.

Il s'agit donc de la création d'un rapport de l'urbain avec les sols et la nature qui associe lieu de villégiature et sécurité alimentaire. En écologie urbaine, les jardins peuvent être définis comme des lieux où s'invente un rapport actif des citadins à une 《 nature produite ${ }^{18}$, pour satisfaire une large gamme de besoins humains. Les jardins, datchas et autres terrains, sont en effet des espaces multifonctionnels dans lesquels la production alimentaire peut être combinée selon les périodes et les besoins des utilisateurs avec des fonctions de repos, de loisir, de récupération de la santé, d'éducation des enfants, de créativité. Cette forme massive de démocratisation de l'accès des urbains à la nature est un élément de flexibilité économique et sociale, un amortisseur des crises, un tampon régulateur.

Une petite partie des jardins et datchas évolue vers le modèle pavillonnaire occidental. Mais la majorité garde les caractéristiques de terrains agricoles qui répondent à une large gamme de besoins humains en préservant et en améliorant les sols. Le statut juridique permettant aux collectifs de jardins, potagers et datchas de ne pas évoluer vers le modèle pavillonnaire en répondant aux besoins de sécurité alimentaire et de villégiature des familles urbaines reste à préciser.

18. Le concept est de Nathalie Blanc (Grenelle de l'Environnement, 2009). 


\section{RÉFÉRENCES BIBLIOGRAPHIQUES}

Alasheev S., Clarke S., Karelina M., Varshavskaya L. (2000). The Myth of Urban Peasant. Work, Employment and Society, vol. 14, n 3, p. 481-499.

Bassine M. Z. (1958). Les jardins des ouvriers et des fonctionnaires. Moscou, Éditions du ministère de l'Agriculture d'URSS, 253 p.

Baudelet L. (2009). Le jardinage urbain : un point presque aveugle de l'appareil statistique français. Paris, FMSH, Réseau international «Développement durable des villes : le rapport entre l'urbain et la nature. Compte-rendu de Séminaire, 14 mai, p. 12-14. http://www.mshparis.fr/recherche/thematiques/developpement-durable-des-villes/

Bertaux D. (1997). Les récits de vie - Perspective ethnosociologique. Paris, Nathan, $128 \mathrm{p}$.

Boukharaeva L. M., Marloie M. L'apport du jardinage urbain de Russie à la théorisation de l'agriculture urbaine. In: Vertig'O, 2010, 10 (2) [en ligne: http://vertigo.revues.org/9919].

Chevery C., Gascuel C. (2009). Sous les pavés, la terre. Montreuil, Omniscience, $208 \mathrm{p}$.

Clarke S. (2002). Making Ends Meet in Contemporary Russia. Secondary Employment, Subsidiary Agriculture and Social Networks. Cheltenham, Edward Elgar, $281 \mathrm{p}$.

Giroux A. (1995). L'agroalimentaire russe en panne entre réforme et conservatisme. Paris, Le Courrier des pays de l'Est, $\mathrm{n}^{\circ} 405$, décembre, p. 3-24.

Giroux A. (1999). L'agriculture russe à l'épreuve de la crise. Paris, Le Courrier des pays de l'Est. $\mathrm{n}^{\circ} 441$, juillet, p. 48-61. Grenelle de 1'Environnement. (2009). Restaurer et valoriser la nature en ville Vers un plan d'action dès 2010. Synthèse de la conférence de lancement du 29 juin. h t t p : / / w w w . l e g r e n e 11 e environnement.fr/grenelle-environnement/spip.php?article1271

Hervé J-J. (2001). Russie. Agriculture : la grande transformation. Compte-rendu de l'Académie d'Agriculture de France. Paris, Problèmes économiques, $\mathrm{n}^{\circ} 2.701$, 21 février, p. 17.

Hervouet R. (2006). L'économie du potager en Biélorussie et en Russie. Paris, Études rurales. $\mathrm{n}^{\circ} 177,2006 / 1$, p. 25-42.

Jogolena H., Kovalenko V. (1995). Les incertitudes de la réforme agraire en Russie. Paris, Revue d'études comparatives Est-ouest, vol. 26, $\mathrm{n}^{\circ}$ 3, septembre, p. 197-227.

Édition des lois fédérales des 22.11.2000 N 137-LF, от 21.03.2002 N 31- LF, от 08.12.2003 N 169-LF, OT 22.08.2004 N 122-LF, от 02.11.2004 N 127-LF, OT 30.06.2006 N 93-LF, от 26.06.2007 N 118- LF. Loi fédérale (1993) : Les unions non commerciales citoyennes des jardins, des potagers et des datchas.

Loi fédérale $\mathrm{n}^{\circ} 93$ du 30 juin (2006). Introduction des changements dans les actes législatifs de la Fédération de Russie sur la question d'un enregistrement simplifié des droits des citoyens à la propriété des biens immobiliers.

Lowell S. (2003). Summerfolk 1710-2000 A History of the Dacha. Ithaca and London, Cornell University Press, 260 p.

Marxer B. (2003). Idéologie foncière en Russie du XVIe siècle à aujourd'hui. Paris, L'Harmattan, $400 \mathrm{p}$.

Ministère du Développement économique de la Fédération de Russie, Agence fédérale du cadastre des biens immobiliers. (2008). Rapport national sur l'état et l'utilisation des terres en Fédération de Russie en 2007. Moscou, 274p. 
Naudet J-B. (1997). De Staline à Eltsine, la victoire du "parti de la patate", Le Monde, 28 mai.

OCDE. (1998). Examen des politiques agricoles. Fédération de Russie. Paris, 294 p.

Ortar N. (2005). Les multiples usages de la datcha des jardins collectifs. Anthropologie et sociétés vol. $29, \mathrm{n}^{\circ} 2$, p. 169 185.

Service fédéral de la statistique d'État de la Fédération de la Russie (2001-2009).

Annuaires statistiques 2001-2009.

Service fédéral de la statistique d'État de la Fédération de la Russie (2008).

Résultats du Recensement agricole de Russie de 2006. Moscou, Centre d'information et d'édition «La statistique de la Russie », 9 vol.

Smith J., Ratta A., Nasr J. (1996). Urban Agriculture: Food, Jobs and Sustainable Cities. New York: United Nations Development Programme, 302 p.
Southworth C. (2006). The Dacha Debate. Household Agriculture and Labor Markets in Post-Socialist Russia. Rural Sociology, vol. 71, n³, p. 451-478.

Traven V. (2005). La datcha en Russie de 1917 à nos jours. Paris, Éd. du Sextant, $232 \mathrm{p}$.

United Nations Division for Sustainable Development. (2000). Sustainable Development Success Stories - Russian Sustainable Agriculture Extension Center. www.un.org/esa/sustdev/groups/success/2 000/SARD-13.htm

Vos six ares. (1991 à 2010). Revue de l'Union des jardiniers. Moscou, Maison de la presse russe.

Zavisca J. (2003). Contesting Capitalism at the Post-Soviet Dacha : The Meaning of Food Cultivation for Urban Russians. Slavic Review, vol. 62, n4, hiver, p. 786810. 